\title{
PARTICLE REMOVAL REACTIONS WITH DEFORMED PROJECTILES
}

\author{
Alexander Sakharuk ${ }^{1}$, P. Gregers Hansen ${ }^{1,2}$, and Vladimir Zelevinsky ${ }^{1,2}$ \\ ${ }^{1}$ National Superconducting Cyclotron Laboratory and \\ ${ }^{2}$ Department of Physics and Astronomy, \\ Michigan State University, East Lansing, MI 48824-1321 USA
}

(November 16, 2018)

Single-particle removal reactions are becoming an important tool for studying radioactive nuclei. The nuclei far from stability may reveal new regions of large deformation. We discuss the influence of the projectile deformation on the stripping cross sections and longitudinal momentum distributions of the core. The Glauber formalism for describing such reactions is developed and compared with the simpler geometric approach and with existing experimental data. The most significant effects are expected for nuclei with valence spherical orbitals strongly mixed by deformation. The shape of the momentum distribution, in turn, can be used for determining the degree of deformation of the projectile.

\section{INTRODUCTION}

Particle removal reactions of various types (nucleon transfer, knockout, stripping and so on) have for a long time been one of the most powerful tools for investigation of nuclear structure [1.2]. We are here concerned with a class of reactions at high energies ("stripping" in the sense of the word used originally by Serber [3]) of which the deuteron breakup is the archetype. The strong current interest in these reactions is that they, in inverse kinematics, provide a unique opportunity to obtain simultaneously partial probabilities for populating individual final states leading to spectroscopic factors as well as momentum distributions characteristic of the quantum numbers of the removed nucleon. This allows one to access 
detailed information on the internal structure of projectile and residue.

Nuclear breakup reactions are widely used nowadays for studying radioactive nuclei [4 6,8]. A new method was suggested recently for the spectroscopy with radioactive beams [7] which is well suited both for "normal" and for halo nuclei. The method uses the longitudinal momentum distribution of the projectile residue ("core") for the determination of the orbital angular momentum of the stripped nucleon. The identification is possible via the comparison of the experimental distribution with the theoretical one calculated, for instance, in the framework of the Glauber model [9,10]. The association of the definite orbital momentum value with the shape of the longitudinal momentum distribution is clearly justified for spherical nuclei. First, for a spherically symmetric unpolarized projectile, the role of the spin-orbit interaction is not essential for the stripping mechanism. Second, the longitudinal momentum distribution is sensitive to the projection $m$ of the orbital momentum of the stripped particle onto the beam direction. As a rule, the largest of the possible $m$-values for a given $l$ value dominates, and this $l$ - and $m$-dependence allows one to determine the orbital momentum more or less uniquely [11]. The results of a recent series of experiments with spherical or weakly deformed nuclei [7] confirm the applicability and flexibility of the method.

Recently the first attempt has been made to apply the same techniques to strongly deformed projectiles. The inclusive reaction ${ }^{9} \mathrm{Be}\left({ }^{25} \mathrm{Al},{ }^{24} \mathrm{Mg}\right) \mathrm{X}$ was investigated in [7]. Many neutron-rich nuclei, for example neon and magnesium isotopes in the region $N \approx 30$ (the island of inversion [12]), are expected to be strongly deformed, and the role of intruder configurations, such as the [3301/2] level coming from the $p f$-shell, is extremely important [13]. This follows from the theoretical calculations [14, 15] and from the results of the latest measurements of the transition probabilities $B\left(E 2 ; 0_{g . s .}^{+} \rightarrow 2^{+}\right)$in neutron-rich ${ }^{26,28} \mathrm{Ne}$ and ${ }^{30,32,34} \mathrm{Mg}$ isotopes via intermediate-energy Coulomb excitation [16]. Available information on the structure of the nuclei in the inversion island is scanty, and one can hope to extract the shape parameters from the analysis of the stripping reactions and corresponding momentum distributions. 
However, in order to correctly interpret experimental data for the deformed nuclei, one needs to modify essentially existing theoretical approaches. Several features of nuclear deformation should be taken into account consistently in the reaction description: (i) the deformed mean field of the core is responsible for the strong mixing of different orbital momenta of the stripped valence nucleon; (ii) although in the case of axial symmetry the projection of the nucleon total angular momentum on the symmetry axis is still a good quantum number (usually denoted as $\Omega$ ), the energy splitting and therefore difference in the population of different doubly degenerate $( \pm \Omega)$ levels preclude complete averaging and stress the effects of the spin-orbit coupling; (iii) pure intrinsic $\Omega$-states of the nucleon have no definite angular momentum projection in the laboratory reference frame defined by the beam direction. All mentioned elements are well known but we have to incorporate them consistently in the reaction formalism. The realistic calculations along these lines imply cumbersome and time consuming numerical work. This might be one of the reasons why, in spite of the growing interest in reactions with deformed nuclei [17, 18], our attempt is apparently the first one of this kind.

Of course, scattering by nonspherical nuclei has been studied long ago. The most general formalism for this purpose is the coupled channel method outlined in the book [19]. Unfortunately, it is rather hard to use this technically complicated method for the description of realistic reactions. A considerably simpler approach, namely the adiabatic approximation, was proposed by Drozdov and Inopin in the middle of the fifties [20,21]. They used the partial wave expansion of the scattering wave function and assumed that the collective motion (rotation or vibration) of the deformed target nucleus is frozen during the collision time, being very slow compared to other degrees of freedom. The term "adiabatic" refers to the orientational and shape degrees of freedom only and should not be confused with the adiabaticity with respect to valence nucleon motion. The latter assumption is also frequently used in Glauber theory for the description of the reaction mechanism. The Glauber adiabaticity means that, starting at energies of 50-100 MeV per nucleon, the characteristic time scales for the relative projectile-target motion and for the nucleon internal motion inside the 
projectile are getting very different.

In the framework of the orientational adiabatic approximation, it is impossible to examine the deep excitations of the core. At the same time, soft excitations, with no essential reorganization of the internal core structure, are quite treatable. Typical energies of collective rotational states are low [19] compared to characteristic reaction energies. In some sense we have here nearly the same situation as in perturbation theory for close or degenerate states. To be consistent with the adiabatic approximation, we fix the orientation of interacting objects during the collision. The state with a certain orientation is a wave packet of stationary angular momentum states. This means that after the collision various rotational members of the superposition can be excited.

At the end of the seventies, Abgrall et al. 222] combined the coupled channel formalism with the Glauber scattering theory to describe the excitation of the ground state rotational band by medium energy $(1 \mathrm{GeV})$ protons. The regular treatment of the adiabatic approximation in the framework of the Glauber theory was given in 1990 by Fäldt and Glauber [23]. They treated only soft rotational excitations without restructuring of the internal projectile wave function. The deformation effect on the stripping cross section was studied only for the relativistic deuteron stripping [24] (see also [25] for a qualitative discussion of the deformation effect on the tensor analyzing power for light projectiles). The approach of our work, being a natural continuation of the guideline [23], is, on the other hand, close to the formulation suggested in the recent paper by Christley and Tostevin [18. These authors came to a conclusion that the deformation is not very important for the total (angle-integrated) reaction cross sections: the corresponding change is less than 1\%. Our results are consistent with this conclusion. However, the longitudinal momentum distributions and partial cross sections for stripping from deformed orbitals, which are a strong mixture of spherical single-particle states, are sensitive to deformation.

The paper is organized as follows. The semi-microscopic reaction formalism, taking into account the deformation of the projectile, is developed in Sec. П]; the influence of the deformation on the stripping reaction is analyzed, and expressions for the cross section and 
the longitudinal momentum distribution of the projectile residue are obtained. Sec. III is devoted to the study of the deformation effects on the core longitudinal momentum distribution in the inclusive, with respect to the rotational states of the core, formulation. This study is performed using a geometric approach [9] which is simpler and more transparent

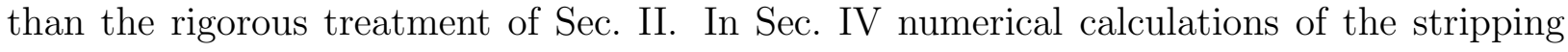
cross sections and longitudinal momentum distributions are presented and discussed in the framework of the approaches of Sec. II and Sec. III for different final states of the residual nucleus in a typical reaction ${ }^{9} \mathrm{Be}\left({ }^{25} \mathrm{Al},{ }^{24} \mathrm{Mg}\right) \mathrm{X}$. The interplay of the internal structure of the projectile and the reaction mechanism is illustrated for the model ${ }^{9} \mathrm{Be}\left({ }^{28} \mathrm{P},{ }^{27} \mathrm{Si}\right) \mathrm{X}$ reaction. We discuss the perspectives for further studies and propose new experiments in a possible region of large deformation in neutron-rich $\mathrm{Mg}$ isotopes. Summary and final remarks are made in the concluding section.

\section{STRIPPING CROSS SECTION FOR A DEFORMED PROJECTILE}

In the description of projectile breakup reactions, the established terminology is slightly different from that conventionally used in the theory of direct reactions [1]. Two processes are usually distinguished, stripping (or absorption) and diffraction (or elastic breakup). In this word usage (see, for example, [26,27]), elastic breakup is the process in which the projectile core and the valence, in particular halo, particle are in the continuum states after the interaction with the target. In addition, the projectile residue (core) and the target remain in their respective ground states. We generalize this definition to include possible "soft" excitations of the core nucleus, such as collective rotations, which disturb the intrinsic structure in a minimal way. The absorption differs from the elastic breakup by possible excitations of the core and disappearance of the valence particle in the final state due to the absorption by the target. In this work we restrict overselves by the treatment of the stripping/absorption type of breakup reactions only. This is, generally, the dominant channel [8], except for the halo states. Before considering the reaction in detail, let us agree 
on a general framework of the approach.

Target and core nuclei are treated as structureless particles in the actual calculations. All the observables depend on the internal structure of the core and the target only indirectly via corresponding interaction potentials and density functions. Nevertheless, we retain the explicit dependence on the internal Jacobi coordinates of the colliding nuclei in the derivation of the basic formulas. This makes the derivation more transparent and shows the way for extending the approach. In addition, the core may be deformed, in contrast to previous treatments 26,28,10]. Therefore we need to fix its orientation (more precisely, the orientation of the core mean field which determines the geometry of the orbitals for the valence nucleon). Although we assume here that the core is axially symmetric, the approach can be generalized for triaxial deformation that would be more suitable for nuclei such as, for example, ${ }^{24} \mathrm{Mg}$. In a similar way, the deformation of the target can be accounted for as would be necessary for transfer reactions.

The interaction of a valence nucleon (for definiteness, a proton) with the target, $\mathrm{p}+\mathrm{T}$, and the core-target, $\mathrm{C}+\mathrm{T}$, interaction are described in terms of phenomenological optical potentials. For the $\mathrm{C}+\mathrm{T}$ interaction, the convolution of a nucleon-target phenomenological potential with the core density is used. We do not employ the traditional Glauber approach based on the nucleon-nucleon scattering amplitudes [29,30 because of relatively small kinetic energy per nucleon in the projectile in the experiments we try to describe. It is known, however, that the region of validity of the Glauber approximation extends considerably beyond what one could expect from the approximations made in the standard derivation [10]. Therefore, our approach can turn out to be very similar to that of Glauber.

The calculations are performed either in the "laboratory" frame, with the origin coinciding with the projectile center-of-mass $O$ and $z$-axis along the beam direction, or in the body-fixed frame, characterized by the origin at the core $O^{\prime}$ and $z^{\prime}$-axis along the core symmetry axis. The positions of the target in these frames are given by the vectors $\mathbf{R}$ and $-\mathbf{r}_{c}$, respectively. The set of coordinates used in our calculations is shown in Fig. 11. 


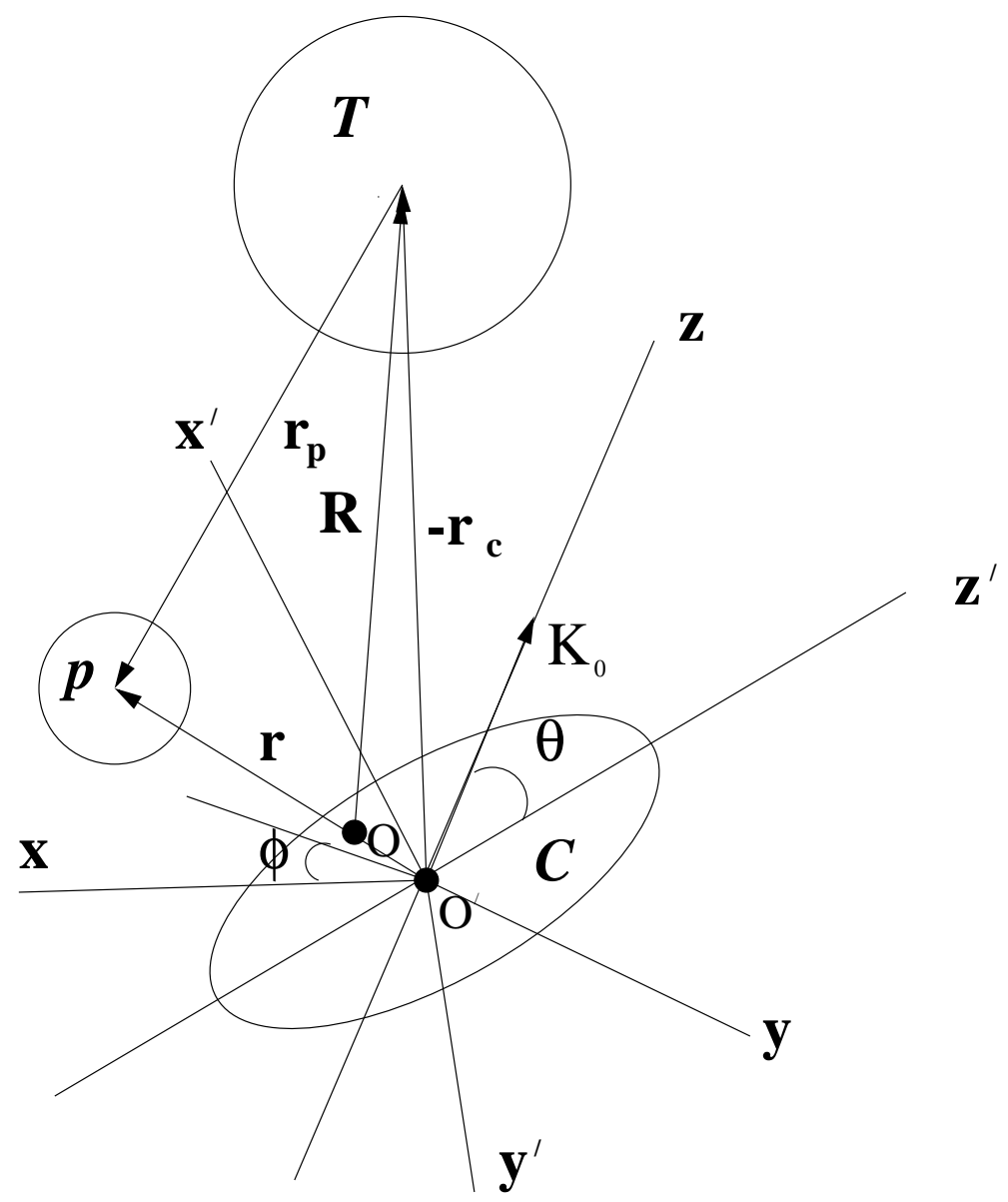

FIG. 1. Coordinates used for the description of the reaction.

The description of stripping reactions with exotic nuclei has its roots in the early works on the deuteron stripping [3,31]. Attempts to construct a more regular theory of stripping processes have appeared considerably later [27,32,33. Referring to the deuteron stripping reaction formalism as a suitable framework for exotic nuclei fragmentation [34], it is assumed by analogy that a stripped projectile constituent (proton) interacts strongly with the target nucleus. The core of the projectile escapes as an analog of the neutron. The only difference is the possibility for the core to be excited during the collision. The total stripping cross section in such an approach is analogous to the proton stripping from the deuteron [2]. Below we sketch briefly the formal derivation of the total stripping cross section and the core longitudinal momentum distribution.

The projectile (core + valence nucleon) initial wave function is 


$$
\left|\chi_{i}\right\rangle=e^{-i \mathbf{K}_{0} \cdot \mathbf{R}}\left|\phi_{i}\right\rangle,
$$

where the plane wave describes motion of the center-of-mass with respect to the target, and the initial intrinsic wave function of the projectile

$$
\left|\phi_{i}\right\rangle=\sum_{\nu \alpha} \mathcal{A}_{\nu}^{(\alpha)} a_{\nu}^{\dagger} \Phi_{\nu}^{(\alpha) \dagger}|0\rangle
$$

is a sum of antisymmetrized products of the core internal wave functions generated by the operator $\Phi_{\nu}^{(\alpha) \dagger}$ and the function of the relative proton-core motion (creation operator $a_{\nu}^{\dagger}$ ). Here the superscript $\alpha$ enumerates various core states, $|\nu\rangle$ are single-particle orbitals, and $\mathcal{A}_{\nu}^{(\alpha)}$ are corresponding spectroscopic amplitudes. The calculation of the spectroscopic amplitudes for all possible combinations $(\nu \alpha)$ requires very specific and hard work. Frequently only one possibility is allowed or is taken into account, which corresponds to a definite configuration, for example to the ground state of the spherical (deformed) core + the proton occupying a certain spherical (deformed) orbital. This approximation works fairly well in the case of light or halo projectiles when there are no bound excited states. The situation is more complicated for projectile nuclei which possess many particle-bound states. We limit ourselves by using the same approximation with the spectroscopic amplitudes calculated in the framework of the particle-rotor model, consistent with our general approach, or borrowed from the shell-model calculations [36]. In the case of a deformed core, the internal wave function contains information about the orientation of the core symmetry axis with respect to the space-fixed frame (the corresponding spherical harmonic $Y_{L_{i} M_{L_{i}}}\left(\Omega_{a}\right)$ ), and the single-particle quantum states $|\nu\rangle$ are quantized onto the intrinsic symmetry axis.

The situation with the final state wave function is even more complicated because here we have to sum over all possible final configurations of the core and valence proton, including the ones where the knocked out proton is absorbed by the target. We do not consider the exact structure of this wave function. Instead we determine [2,31] the absorption cross section through a deficit of the probability for the proton to be found at some point $\mathbf{r}_{p}$ that appears as a result of the $\mathrm{p}+\mathrm{T}$ interaction. Such an approach is based essentially on the spectator mechanism of the stripping reaction and is adequate only in a narrow kinematic 
region. This mechanism presupposes that the proton is stripped off the projectile while the remaining core fragment continues its motion essentially without being disturbed except for a smooth action of the optical core-target potential $V_{\mathrm{C}+\mathrm{T}}$ taken into account by the eikonal core phase shift. The incident energy of the projectile has to be sufficiently large and the scattering angle of the core must be small to ensure the applicability of the eikonal approximation [27,32]. It is assumed in the framework of the spectator mechanism that the nucleus-nucleus interaction time is too short for a change of the core symmetry axis direction. As we mentioned, this means the possibility to reveal various rotational components of the frozen orientation. Therefore the final-state deformed core wave function $\Phi_{\nu}^{(\alpha) \dagger}|0\rangle$ contains a spherical harmonic $Y_{L_{f} M_{L_{f}}}\left(\Omega_{a}\right)$, which defines the same direction of the core symmetry axis as before the projectile breakup (adiabatic approximation), but in general with $L_{f}, M_{f} \neq$ $L_{i}, M_{i}$.

According to the idea of the Glauber approximation and our hypotheses of the reaction mechanism, the reaction yield is determined by the profile functions for the propagation of the valence proton, $S_{p}$, and the core, $S_{c}$, in the field of the target potentials, $\mathrm{p}+\mathrm{T}$ and $\mathrm{C}+\mathrm{T}$, respectively [26],

$$
\begin{aligned}
& S_{p}\left(\mathbf{b}_{p}, z_{p}\right)=\exp \left(-\frac{i}{v} \int_{-\infty}^{z_{p}} V_{\mathrm{p}+\mathrm{T}}\left(\mathbf{b}_{p}, z_{p}^{\prime}\right) d z_{p}^{\prime}\right), \\
& S_{c}\left(\mathbf{b}_{c}, z_{c}\right)=\exp \left(-\frac{i}{v} \int_{-\infty}^{z_{c}} V_{\mathrm{C}+\mathrm{T}}\left(\mathbf{b}_{c}, z_{c}^{\prime}\right) d z_{c}^{\prime}\right) .
\end{aligned}
$$

Here and below we have adopted units with $\hbar=1$. The coordinates $\mathbf{r}_{p}$ and $\mathbf{r}_{c}$ are expressed from Fig. 1 as

$$
\mathbf{r}_{p}=\frac{A_{c}}{A_{c}+1} \mathbf{r}-\mathbf{R}, \quad \mathbf{r}_{c}=-\frac{1}{A_{c}+1} \mathbf{r}-\mathbf{R} .
$$

The impact parameter vectors $\mathbf{b}_{p}$ and $\mathbf{b}_{c}$ are their corresponding two-dimensional projections onto the plane perpendicular to the beam direction.

In the same spirit, the (core + proton) Glauber wave function in the final state, projected onto definite states of core and proton motion with momenta $\mathbf{k}_{c}$ and $\mathbf{k}_{p}$, respectively, is 


$$
\left|\chi_{f}^{(-)}\right\rangle=\tilde{S}_{p}\left(\mathbf{b}_{p}, z_{p}\right) \tilde{S}_{c}\left(\mathbf{b}_{c}, z_{c}\right) e^{i \mathbf{k}_{c} \cdot \mathbf{r}_{c}} e^{i \mathbf{k}_{p} \cdot \mathbf{r}_{p}} \Phi_{\nu}^{(\alpha) \dagger}|0\rangle
$$

where

$$
\tilde{S}_{p}\left(\mathbf{b}_{p}, z_{p}\right)=\exp \left(\frac{i}{v} \int_{z_{p}}^{\infty} V_{\mathrm{p}+\mathrm{T}}\left(\mathbf{b}_{p}, z_{p}^{\prime}\right) d z_{p}^{\prime}\right)
$$

and

$$
\tilde{S}_{c}\left(\mathbf{b}_{c}, z_{c}\right)=\exp \left(\frac{i}{v} \int_{z_{c}}^{\infty} V_{\mathrm{C}+\mathrm{T}}\left(\mathbf{b}_{c}, z_{c}^{\prime}\right) d z_{c}^{\prime}\right)
$$

Here we again take into account the interactions with the target by means of the eikonal phase shifts [32.

The stripping reaction is defined essentially as disappearance of the proton from the projectile as a result of the collision with the target. We need to compare the probabilities for the proton to be found at some point $\mathbf{r}_{p}$ with and without the $\mathrm{p}+\mathrm{T}$ interaction. The probability amplitude for the core in a given configuration $\alpha$ to have the momentum $\mathbf{k}_{c}$ and for the valence proton, emerging from the intrinsic state $|\nu\rangle$ with the spectroscopic amplitude $\mathcal{A}_{\nu}^{(\alpha)}$, to be found at the position $\mathbf{r}_{p}$ is

$$
a\left(\mathbf{k}_{c}, \mathbf{r}_{p}\right)=\mathcal{A}_{\nu} S_{p} e^{-i \mathbf{k}_{p} \cdot \mathbf{r}_{p}} \int d^{3} r_{c} e^{-i \mathbf{k}_{c} \cdot \mathbf{r}_{c}+i \mathbf{K}_{0} \cdot \mathbf{R}_{\phi_{\nu}}(\mathbf{r})\left\langle 0\left|\Phi_{\nu} S_{c} \Phi_{\nu}^{\dagger}\right| 0\right\rangle},
$$

where $\mathbf{r}=\mathbf{r}_{p}-\mathbf{r}_{c}, \phi_{\nu}(\mathbf{r})=\left\langle\mathbf{r}\left|a_{\nu}^{\dagger}\right| 0\right\rangle$ is the function of the relative proton-core motion in the projectile nucleus, and we omit the arguments of the profile functions as well as the superscript $\alpha$. The phase integrals in the profile functions $S_{c}$ and $S_{p}$ in (8) are now taken from $-\infty$ to $\infty$ and do not depend on the $z$-components of the core and proton radius-vectors. The intrinsic matrix element in (\$) and the profile function $S_{c}$ contain the orientation parameters of the deformed core which are adiabatically fixed during the collision. Therefore the whole amplitude $a\left(\mathbf{k}_{c}, \mathbf{r}_{p}\right)$ is still an operator in orientation angles. Thus, apart from the stripping probability, it will determine the rotational excitations of the remaining core.

The core final momentum is a sum of $\left(A_{c} / A\right) \mathbf{K}_{0}$ (the core part of the initial momentum of the projectile as a whole) and the internal momentum q corresponding to relative coreproton motion inside the projectile. Due to the large mass difference, $A_{c} \gg A_{p}$, we can put $A_{c} \approx A$ and obtain for the exponent in the integrand (8) 


$$
-i \mathbf{k}_{c} \cdot \mathbf{r}_{c}-i \mathbf{K}_{0} \cdot \mathbf{R}=-i \mathbf{q} \cdot \mathbf{r}_{c}
$$

Eq. (8) thus can be rewritten as

$$
a\left(\mathbf{k}_{c}, \mathbf{r}_{p}\right)=\mathcal{A}_{\nu} S_{p} e^{-i \mathbf{k}_{p} \cdot \mathbf{r}_{p}} \int d^{3} r_{c} e^{-i \mathbf{q} \cdot \mathbf{r}_{c}} \phi_{\nu}(\mathbf{r})\left\langle 0\left|\Phi_{\nu} S_{c} \Phi_{\nu}^{\dagger}\right| 0\right\rangle
$$

Without the $\mathrm{p}+\mathrm{T}$ interaction, the expression (9) reduces to

$$
a_{0}\left(\mathbf{k}_{c}, \mathbf{r}_{p}\right)=\mathcal{A}_{\nu} e^{-i \mathbf{k}_{p} \cdot \mathbf{r}_{p}} \int d^{3} r_{c} e^{-i \mathbf{q} \cdot \mathbf{r}_{c}} \phi_{\nu}(\mathbf{r})\left\langle 0\left|\Phi_{\nu} S_{c} \Phi_{\nu}^{\dagger}\right| 0\right\rangle
$$

The probability for the proton to get absorbed in different spatial points $\mathbf{r}_{p}$, provided that the core escapes with the momentum $\mathbf{k}_{c}$, is proportional to the difference of the squares of the absolute values of the orientational matrix elements of the operators (10) and (9) between the given rotational states of the core,

$$
\begin{gathered}
\left|a_{0}\left(\mathbf{k}_{c}, \mathbf{r}_{p}\right)\right|_{f i}^{2}-\left|a\left(\mathbf{k}_{c}, \mathbf{r}_{p}\right)\right|_{f i}^{2}=\left|\mathcal{A}_{\nu}\right|^{2}\left(1-\left|S_{p}\left(\mathbf{b}_{p}\right)\right|^{2}\right) \\
\times\left|\int d^{3} r_{c} e^{-i \mathbf{q} \cdot \mathbf{r}_{c}} \int d \Omega_{a} \phi_{\nu}(\mathbf{r}) Y_{L_{f} M_{L_{f}}}^{*}\left(\Omega_{a}\right) S_{c}\left(\mathbf{b}_{c}\right) Y_{L_{i} M_{L_{i}}}\left(\Omega_{a}\right)\right|^{2} .
\end{gathered}
$$

Here we have explicitly written the arguments of the profile functions and preserved only those parts of the core wave functions that define the orientation of the core mean field. In the integrand we have the product of four factors: two spherical harmonics defining the core rotational state before and after the interaction with the target, the core profile function (our operator), and the function of the proton-core relative motion $\phi_{\nu}(\mathbf{r})$. The dependence of $\phi_{\nu}(\mathbf{r})$ upon the core orientation is considered in detail in the next section.

The approach above ensures the correct limit of spherical symmetry. Indeed, in this case all factors in (11), except the spherical functions, do not depend on the orientation angles $\Omega_{a}$, and the orthonormalization of the spherical harmonics singles out the diagonal transition of the core. Another way to consider the core orientation would be to retain the spherical harmonics up to the final calculation of the stripping differential/total cross section and then average over all possible directions of the core symmetry axis. This would however imply ignoring interference contributions between the processes of rotational recoil 
of the core occurring at different positions of the core. In addition, in that case we encounter a technical problem of summation over all possible final projections $M_{L_{f}}$ (final channels). Since the values of $M_{L_{f}}$ are connected with the orientation of the core axis $\Omega_{a}$, the simple summation can lead to the double counting.

The differential cross section for the core final momentum $d^{3} k_{c}=d^{3} q$ is obtained, as in the deuteron stripping theory [2], after integrating the difference of probabilities (11) on the interaction plane, orthogonal to the projectile direction, over the proton impact parameter $\mathbf{b}_{p}$

$$
\begin{gathered}
\frac{d \sigma}{d^{3} q}=\frac{1}{(2 \pi)^{3}} \bar{\sum}\left|\mathcal{A}_{\nu}\right|^{2} \int d^{2} b_{p}\left(1-\left|S_{p}\left(\mathbf{b}_{p}\right)\right|^{2}\right) \\
\times\left|\int d^{3} r e^{-i \mathbf{q} \cdot \mathbf{r}} \int d \Omega_{a} \phi_{\nu}(\mathbf{r}) Y_{L_{f} M_{L_{f}}}^{*}\left(\Omega_{a}\right) S_{c}\left(\mathbf{b}_{c}\right) Y_{L_{i} M_{L_{i}}}\left(\Omega_{a}\right)\right|^{2},
\end{gathered}
$$

where $\bar{\sum}$ means, as usual, the averaging over the initial angular momentum projections and summation over the final ones. Here we made a change of variable from the set $\mathbf{r}_{p}$ and $\mathbf{r}_{c}$ to the set $\mathbf{r}_{p}$ and $\mathbf{r}=\mathbf{r}_{c}-\mathbf{r}_{p}$. The three-dimensional momentum distribution of the core (12) differs from the results of refs. [26,10] by the presence of the spherical harmonics with the additional integration over $\Omega_{a}$.

The longitudinal momentum distribution of the core is obtained by integrating over the transverse components $\mathbf{q}_{\perp}$,

$$
\begin{gathered}
\frac{d \sigma}{d q_{z}}=\frac{1}{2 \pi} \bar{\sum}\left|\mathcal{A}_{\nu}\right|^{2} \int d^{2} b_{p}\left(1-\left|S_{p}\left(\mathbf{b}_{p}\right)\right|^{2}\right) \\
\times \int d x d y\left|\int d z e^{-i q_{z} z} \int d \Omega_{a} \phi_{\nu}(\mathbf{r}) Y_{L_{f} M_{L_{f}}}^{*}\left(\Omega_{a}\right) S_{c}\left(\mathbf{b}_{c}\right) Y_{L_{i} M_{L_{i}}}\left(\Omega_{a}\right)\right|^{2} .
\end{gathered}
$$

The total proton stripping cross section follows after the integration over $q_{z}$,

$$
\sigma=\bar{\sum}\left|\mathcal{A}_{\nu}\right|^{2} \int d^{2} b_{p}\left(1-\left|S_{p}\left(\mathbf{b}_{p}\right)\right|^{2}\right) \int d^{3} r\left|\int d \Omega_{a} \phi_{\nu}(\mathbf{r}) Y_{L_{f} M_{L_{f}}}^{*}\left(\Omega_{a}\right) S_{c}\left(\mathbf{b}_{c}\right) Y_{L_{i} M_{L_{i}}}\left(\Omega_{a}\right)\right|^{2}
$$

The expression obtained in a number of works [26,10] differs by the absence of the angular integral. In fact, these results are known from the theory of deuteron stripping [2, 31], of 
course again without deformation effects. The more complicated and formal approaches, such as, for example, based on Ref. [27], do not bring any real advantage.

The presence of the angular integral in (13, 14), together with the angular dependence of the core profile function $S_{c}\left(\mathbf{b}_{c}\right)$ and, especially, the proton wave function $\phi_{\nu}(\mathbf{r})$, makes a practical calculation of the longitudinal momentum distribution (13) and the total stripping cross section (14) a difficult computational problem. Eq. (14), for example, contains seven embedded integrals. In addition, the function $\phi_{\nu}(\mathbf{r})$ and its arguments depend on the angular variables in a very complicated way. To ensure the resonable accuracy one needs to carefully check the convergence of each numerical integration. In practice we have used a time consuming combination of the grid integration with the Monte Carlo methods. We guess that these technical problems were and remain up to now the main obstacle preventing the consistent account of the deformation effects in the calculation of reaction observables.

\section{III. “GEOMETRIC" DESCRIPTION: LONGITUDINAL MOMENTUM DISTRIBUTION OF THE CORE}

The longitudinal momentum distribution may be calculated directly from (13). Technically, this is very time-consuming. Indeed, we need to perform a multidimensional integration over single-particle and core coordinates and over the orientational angles $\Omega_{a}$. Prior to that, we have to calculate the core profile function $S_{c}$ as a convolution of the nucleon-target potential with a core density distribution which, in turn, strongly depends on the orientation of the core symmetry axis. To avoid these technical difficulties, one can use a simpler approach developed in [9] on the "physical" level of rigor.

In the approach [9] we assume that the profile functions are step functions ("black disc" approximation). Further, the interaction time is very short (a sudden approximation) so that the core after reaction retains the same momentum distribution as in the projectile before the collision with a target. Due to conservation of the total momentum, the core

momentum distribution is determined by that for the stripped valence nucleon inside the 
projectile. Therefore, one needs to calculate the the three-dimentional Fourier transform of the part of the initial valence nucleon wave function which is cut out by the target. Geometrically the overlapping region of the valence nucleon wave function with the target corresponds [9] to a cylinder with the axis along the beam direction and the radius defined [4] by the effective radius of the target $R_{T}$.

The approach [9] can be extended straightforwardly in order to take into account the projectile deformation. It is easy to include the case of deformed targets also but with significant computational complications. In what follows we consider nucleon stripping from a deformed projectile on a spherical target.

The core mean-field deformation can be treated in the framework of the standard Nilsson model in the projectile body-fixed frame [19,38,39. Double degenerate single-particle orbitals labeled by the asymptotic quantum numbers $\left[N n_{z} \Lambda \Omega\right]$ are obtained by the diagonalization of the phenomenological nuclear Hamiltonian in the basis of spherically symmetric wave functions

$$
\left|\left[N n_{z} \Lambda \Omega\right]\right\rangle=\sum_{l j} \alpha_{l j \Omega}^{\left[N n_{z} \Lambda\right]}|l j \Omega\rangle .
$$

Note that the spin-orbit coupling is essential for the correct definition of single-particle states. For the $s d$-shell nuclei we have six double degenerate basis states $|l j \Omega\rangle$ some of which (with the same angular momentum projection $\Omega$ onto the core symmetry axis) are coupled due to the static core deformation. The corresponding single-particle orbitals in the laboratory frame result from the rotation $\hat{T}(\mathcal{R})$,

$$
\psi(\mathbf{r})=\hat{T}(\mathcal{R})\left|\left[N n_{z} \Lambda \Omega\right]\right\rangle=\sum_{l j} \alpha_{l j \Omega}^{\left[N n_{z} \Lambda \Omega\right]} \sum_{\Omega^{\prime}=-j}^{j} D_{\Omega^{\prime} \Omega}^{j}(\mathcal{R})\left|\operatorname{lj} \Omega^{\prime},\{\hat{\mathcal{R}} \mathbf{r}\}\right\rangle,
$$

where $D_{\Omega^{\prime} \Omega}^{j}(\mathcal{R})$ are elements of the rotation matrix [40]. The operator $\hat{\mathcal{R}}$ is defined by the Euler angles $\theta_{1}, \theta_{2}, \theta_{3}$ but one of these angles is arbitrary in the case of axial symmetry and may be set equal to zero. The remaining two are the polar and azimuthal angles between the core symmetry axis and the beam direction, $\theta_{1}=\varphi, \theta_{2}=\vartheta$.

The three-dimensional nucleon momentum distribution inside the projectile is the squared Fourier transform of the wave function (16) 


$$
\tilde{\psi}(\mathbf{q})=\int d^{3} r e^{-i \mathbf{q} \cdot \mathbf{r}} \psi(\mathbf{r})
$$

For a specific reaction, the momentum distribution corresponds to the spatial integration restricted by the region $G$ that, in the plane perpendicular to the beam $(z)$ direction, is a disk with the center at the point $\left(x=b_{\min }, y=0, z=0\right)$ and the radius $R_{T}$. Here $b_{\text {min }}$ is the lowest possible value of the impact parameter $b_{\text {min }}=R_{c}+R_{T}, R_{c}=r_{o} A_{c}^{1 / 3}$ is the effective core radius (it is supposed that the interactions with the impact parameter less than $b_{\min }$ lead to violent collisions and cannot be seen in the stripping channel). The longitudinal momentum distribution is obtained by integrating $|\tilde{\psi}|^{2}$ over the transverse $\mathbf{q}$ components,

$$
\frac{d W}{d q_{z}}=\int\left|\tilde{\psi}\left(\mathbf{q}_{\perp}, \mathbf{q}_{z}\right)\right|^{2} \frac{d^{2} q_{\perp}}{(2 \pi)^{3}}=\frac{1}{2 \pi} \int_{G} d x d y\left|\int_{-\infty}^{\infty} d z e^{i q_{z} z} \psi(x, y, z)\right|^{2}
$$

Using eq. (16) we obtain

$$
\begin{aligned}
& \frac{d W}{d q_{z}}=\frac{1}{2 \pi} \int_{G} d x d y \mid \int_{-\infty}^{\infty} d z e^{i q_{z} \cdot z} \sum_{l j} \alpha_{l j \Omega}^{\left[N n_{z} \Lambda\right]} \sum_{\Omega^{\prime}=-j}^{j} D_{\Omega^{\prime} \Omega}^{j}(\mathcal{R}) \\
& \times\left.\sum_{\Lambda}\left(l \Lambda, 1 / 2 \Omega^{\prime}-\Lambda \mid j \Omega^{\prime}\right) R_{n l}(r) Y_{l \Lambda}(\hat{\mathbf{r}}) \chi_{1 / 2 \Omega^{\prime}-\Lambda}\right|^{2} .
\end{aligned}
$$

Taking into account the orthogonality of the spin functions $\chi_{1 / 2} \Omega^{\prime}-\Lambda$, after some algebra we obtain the final expression for the longitudinal momentum distribution of the stripped nucleon

$$
\begin{gathered}
\frac{d W}{d q_{z}}=\frac{1}{2 \pi} \int_{G} d x d y \sum_{\sigma= \pm 1 / 2} \mid \sum_{l j} \alpha_{l j \Omega}^{\left[N n_{z} \Lambda\right]} \sum_{\Omega^{\prime}=-j}^{j} D_{\Omega^{\prime} \Omega}^{j}(\mathcal{R})\left(l \Omega^{\prime}+\sigma, 1 / 2-\sigma \mid j \Omega^{\prime}\right) \\
\times\left.\int_{-\infty}^{\infty} d z e^{i q_{z} z} R_{N l}(\hat{\mathcal{R}} r) Y_{l \Omega+\sigma}(\hat{\mathcal{R}} \hat{\mathbf{r}})\right|^{2}
\end{gathered}
$$

The single-nucleon stripping cross section is obtained by integrating over $q_{z}$,

$$
\begin{gathered}
\sigma=\int_{G} d x d y \sum_{\sigma= \pm 1 / 2} \int_{-\infty}^{\infty} d z \mid \sum_{l j} \alpha_{l j \Omega}^{\left[N n_{z} \Lambda\right]} \sum_{\Omega^{\prime}=-j}^{j} D_{\Omega^{\prime} \Omega}^{j}(\mathcal{R})\left(l \Omega^{\prime}+\sigma, 1 / 2-\sigma \mid j \Omega^{\prime}\right) \\
\times\left. R_{N l}(\hat{\mathcal{R}} r) Y_{l \Omega+\sigma}(\hat{\mathcal{R}} \hat{\mathbf{r}})\right|^{2} .
\end{gathered}
$$


The longitudinal momentum distribution (20) and total stripping cross section (21) are obtained at a fixed direction of the core symmetry axis. We assumed that the core can not change its orientation during the interaction time. But we did not impose any other limitations on the core state of motion - the core can be cranked by the process of interaction obtaining any angular momentum allowed by selection rules. Since we do not specify the state of the core exactly we have here an "inclusive" process with respect to the core final states. It means, in turn, that the cross section (21) is to be understood as a sum of partial cross sections corresponding to the excitation of the particular core rotational states and calculated in the framework of the more regular approach of Sec. II where all interference features were fully accounted for. In distinction to that, in the geometric description the stripping processes intitated at different positions of the core are added incoherently and, for the unpolarized projectile, we have to average the $d W / d q_{z}$, eq. (20), over all possible orientational angles $\varphi, \vartheta$. It gives an additional double integration and the factor $1 / 4 \pi$ in $(20,21)$. We do not have any averaging in the approach of Sec. [I] where the integration over the orientational angles appears as a part of the calculation of the matrix element over the core internal wave functions. An attempt to carry out the averaging leads to uncertainties in the summation over core final states (double counting mentioned in Sec. II).

\section{RESULTS AND DISCUSSION}

For practical calculations we use the Nilsson Hamiltonian (with constants from [19]) expressed in the dimensionless stretched coordinates [39]. The coordinate dependence of the basis wave functions in (16) upon the rotation operator noticeably lengthens the computer calculations. The harmonic oscillator wave functions also have an incorrect asymptotic behavior, decaying too fast at large distances. This shortcoming is not very serious for tightly bound projectiles (with the binding energies $\geq 5 \mathrm{MeV}$ ). However for the description of weakly bound nuclei, the realistic behavior of single-particle wave functions may be crucial so that in this case the results obtained with the aid of the Nilsson Hamiltonian should be 
considered as preliminary estimates.

We start with the inclusive reaction ${ }^{9} \mathrm{Be}\left({ }^{25} \mathrm{Al},{ }^{24} \mathrm{Mg}\right) \mathrm{X}$ studied experimentally in [7]. The deformed nature of the ${ }^{24} \mathrm{Mg}$ nucleus [19] and, correspondingly, ${ }^{25} \mathrm{Al}$ projectile, is well established. Our analysis based on the expression (20) can be considerably simplified in the case of the observation of the core nucleus in its ground state. Indeed, the valence proton in ${ }^{25} \mathrm{Al}$ occupies the Nilsson orbital [202 5/2]. This orbital is generated by the spherical $j=5 / 2, m_{j}=5 / 2$ basis function which is not mixed by the core deformed mean field with other spherical orbitals from the $s d$-shell so that we have only a slight deformation dependence through the use of the stretched coordinates.

The longitudinal momentum distributions of the ${ }^{24} \mathrm{Mg}$ nucleus in the intrinsic ground state (frozen orientation) calculated according to (20) for different values of the oscillator deformation parameter $\delta[19,38,39]$ are shown in Fig. 2. The theoretical curves are normalized to the inclusive experimental data, the normalization constants differ for different $\delta$. We stress again that the experimental data are inclusive and represent the sum of differential cross sections to all final states with isospin $T=0$ and $T=1$.

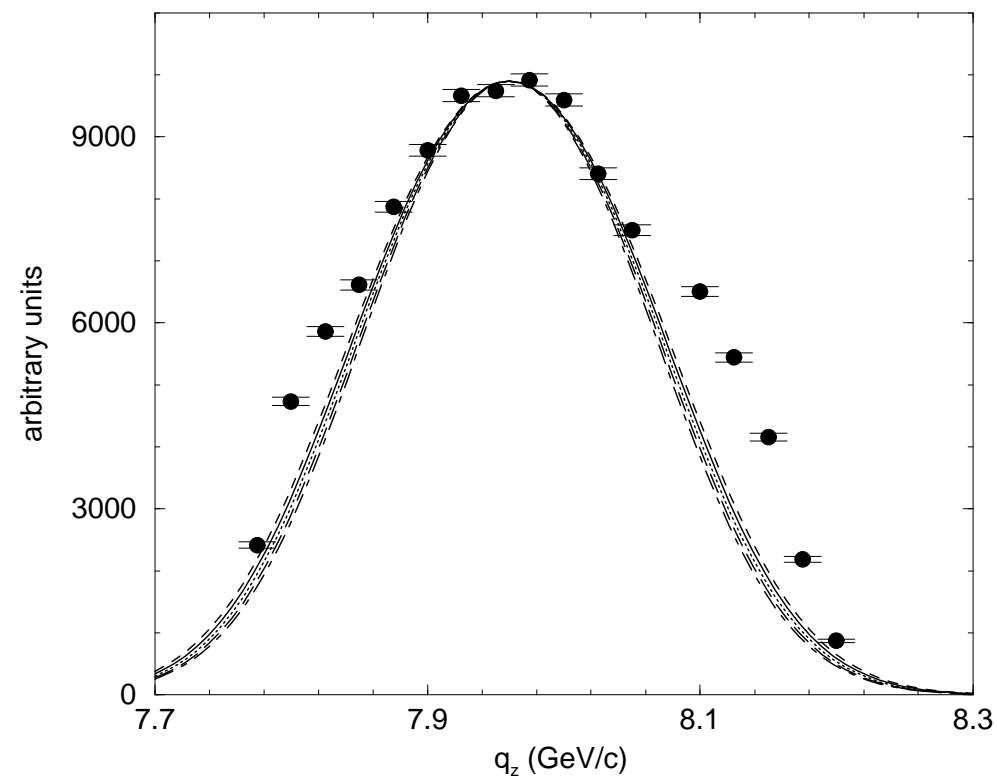


FIG. 2. Longitudinal momentum distributions of ${ }^{24} \mathrm{Mg}$ in the stripping reaction ${ }^{9} \mathrm{Be}\left({ }^{25} \mathrm{Al},{ }^{24} \mathrm{Mg}\right) \mathrm{X}$ with the ${ }^{24} \mathrm{Mg}$ nucleus in the intrinsic ground state. The curves correspond to different values of the deformation parameter: $\delta=0$ (solid), $\delta=0.1$ (dotted), $\delta=0.2$ (dashed), $\delta=0.3$ (long dashed), and $\delta=0.4$ (dash-dotted). The experimental points for the inclusive process, together with the error bars, from [7] are shown. The initial beam energy is $65 \mathrm{MeV}$ per nucleon.

Note that the case $\delta=0$ would not directly correspond to the spherical symmetry of the projectile. Indeed, to achieve such correspondence we have to take into account the degeneracy of three $d_{5 / 2}$ sublevels with $\Omega \rightarrow m_{j}= \pm 5 / 2, \pm 3 / 2$, and $\pm 1 / 2$. In our case we move along the only Nilsson [202 5/2] orbital that corresponds to a single value of $\left|m_{l}\right|=2$ 11] and, of course, can not account for other orbitals. To be consistent with the Nilsson model, we do not draw the momentum distributions for the mixture of different $m_{j}$ states.

The total stripping cross sections obtained from (20) by integration over $q_{z}$ are shown in Table 1. Although the cross section generally decreases with the growth of the deformation, in the region $\delta \sim 0.3-0.5$, where the deformation parameter corresponds to the realistic value $\delta \approx 0.34$ 41] for the ${ }^{24} \mathrm{Mg}$ core, the cross section reaches the minimum and starts to increase again. This can be considered as a hint toward the explanation of the difference between the theoretical cross section calculated in the framework of spherical nuclei and the experimental one. Note again that for a comparison with the case of sperical symmetry we need to take into account the stripping from two additional single-particle orbitals [211 3/2] and $[2201 / 2]$; the corresponding stripping cross sections at $\delta=0$ are equal to 18.6 and 25.28 mb. The standard averaging over the different orbitals gives approximately $21 \mathrm{mb}$ for the cross section corresponding to the absence of the deformation which agrees resonably well with the value of $\sim 26 \mathrm{mb}$, calculated for the spherically symmetric projectile and realistic valence proton wave function (with correct asymptotic behavior). Our cross sections should always be smaller than their counterparts for the functions with correct asymptotics.

In the calculation of the longitudinal momentum distributions and stripping cross sections we have used the following values for the effective target radius and minimum impact 
parameter: $R_{T}=2.948 \mathrm{fm}$ (it corresponds to the cross section $\sigma=273 \mathrm{mb}$ for the free proton interaction with ${ }^{9} \mathrm{Be}$ ) and $b_{\min }=6.094 \mathrm{fm}$.

Table 1. The cross sections, in mb, for the stripping of the [202 5/2] proton from ${ }^{25} \mathrm{Al}$ to the ${ }^{24} \mathrm{Mg}$ ground state rotational band at different deformation parameters $\delta$.

\begin{tabular}{|c|c|c|c|c|c|}
\hline$\delta$ & 0 & 0.1 & 0.2 & 0.3 & 0.4 \\
\hline$\sigma$ & 19.2 & 18.6 & 18.2 & 18.1 & 18.3 \\
\hline
\end{tabular}

Let us now compare these results with the more elaborate calculations based on the regular theoretical approach of Sec. III. We limit ourselves here by the consideration of the realistic ${ }^{24} \mathrm{Mg}$ deformation parameter $\delta \approx 0.34$ [41] under the assumption of axial symmetry. In this approach we can calculate not only the total stripping cross section summed over all core collective rotational states, as was done above (Table 1), but also the partial cross sections for the transitions into different rotational states of the core. If the results of the two rather different approaches are consistent, the inclusive, with respect to rotational states, cross section calculated according to the prescription of Sec. III has to coincide with the sum of all partial cross sections obtained in the framework of the more regular theoretical approach of Sec. [1]. The cross sections based on eq. (14) for the transitions into three lowest levels of the $0^{+}$rotational band of ${ }^{24} \mathrm{Mg}$ are shown in Table 2.

Table 2. The cross sections, in mb, for the stripping of $[2025 / 2]$ proton from ${ }^{25} \mathrm{Al}$ for the three lowest rotational states of the core. The deformation parameter $\delta=0.34$.

\begin{tabular}{|c|c|c|c|c|}
\hline$L_{f}$ & 0 & 2 & 4 & $\geq 6$ \\
\hline$\sigma$ & 7.3 & 13.6 & 1.0 & negligibly small \\
\hline
\end{tabular}


The total cross section is equal to $22 \mathrm{mb}$ that agrees with the inclusive cross section $\approx 18.2$ mb calculated in the framework of the simple approach (see Table 1). The agreement would be even closer if we would take into account the value of the spectroscopic factor $\mathcal{A}_{\nu}^{2}$ in (14). We have estimated the spectroscopic factor using the particle-rotor model for the ${ }^{25} \mathrm{Al}$ nucleus and obtained the total cross section which varies from 20.7 to $21.5 \mathrm{mb}$.

As for the shape of the core longitudinal momentum distribution, using the full calculation of Sec. 【1 we obtain a nearly perfect agreement with experimental data as shown in Fig. 3. The dashed curve corresponds to the transition into the $0^{+}$core ground state, the dashed-dotted one - to the transition into the $2^{+}$state, and the solid curve is the sum of the transitions to $0^{+}, 2^{+}$, and $4^{+}$states. Note that all calculations have been performed without any adjustable parameters except for the overall normalization.

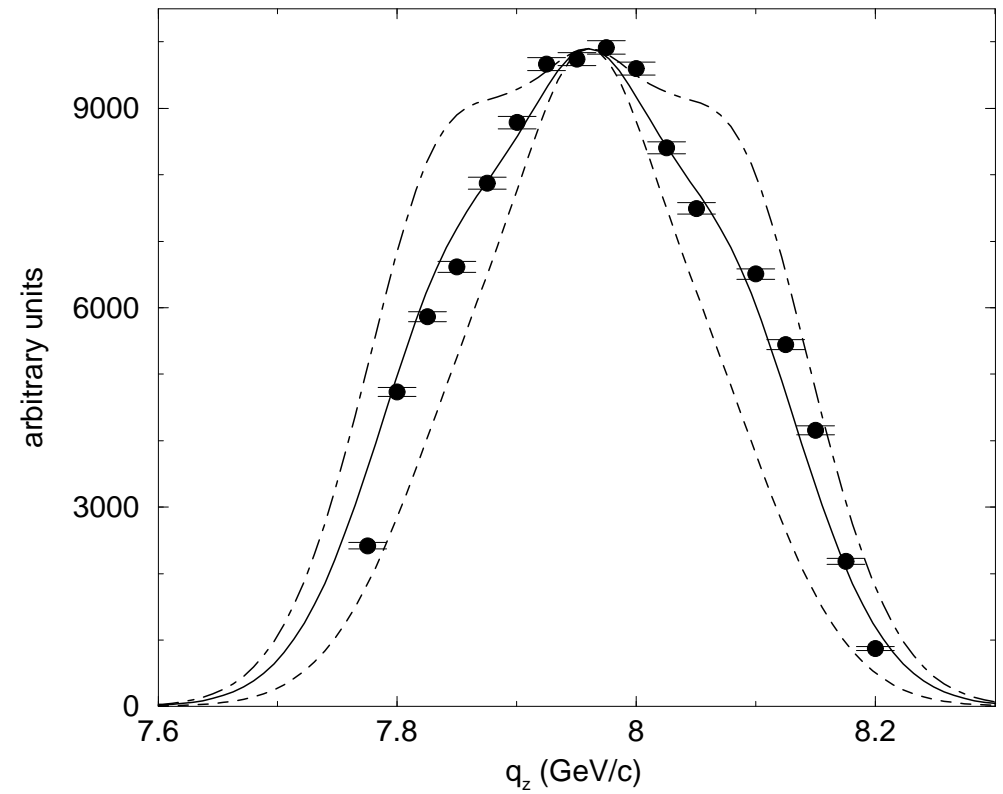

FIG. 3. Longitudinal momentum distributions of ${ }^{24} \mathrm{Mg}$ in the stripping reaction ${ }^{9} \mathrm{Be}\left({ }^{25} \mathrm{Al},{ }^{24} \mathrm{Mg}_{0^{+}, 2^{+}, 4^{+}}\right) \mathrm{X}$. The dashed curve corresponds to the transition into the $0^{+}$core state, the dashed-dotted one - to the transition into $2^{+}$state, and the solid curve is the sum of the three transitions including the weak $4^{+}$(not shown separately). The experimental points together with the error bars from [7] are shown. The initial beam energy is $65 \mathrm{MeV}$ per nucleon.

Fig. 2 and Table 1 seemed to indicate that the deformation is not very important both for 
stripping cross sections and longitudinal momentum distributions. This agrees also with the results of ref. [18]. However, as we see from Fig. 3 and Table 2, such a conclusion would be premature. The details of the longitudinal momentum distributions can be explained only after taking into account the deformation of the projectile. This is clearly seen in Fig. 1 where we compare the longitudinal momentum distributions calculated in the framework of exact theory of Sec. [1], the solid line, in the extended geometric approach of Sec. III, the dotted line, and in the original geometric approach of Ref. [9] developed for the spherical projectiles, the dashed line. The comparison of three approaches made in Fig. 4 with the absolute normalization to the experimental peak is incomplete since the spherical geometric approach, as a rule, leads to the cross sections of considerably reduced magnitude (up to $25 \%)$.

Three features are worth pointing out in connection with the comparison in Fig. 4 . The first is that although, as noted earlier in our paper, the use of harmonic-oscillator wave functions leads to a wrong asymptotic behavior at large distances, this problem should be a minor one here. The reason for this is the relatively large separation energy for the proton combined with the effect of the Coulomb barrier, both making the tail of nucleon wave function unimportant. Therefore the comparison with the calculation [7], which used a $d_{5 / 2}$ wave function from a spherical Woods-Saxon potential with Coulomb interaction included, can be expected to be quantitatively meaningful. In this connection we note, secondly, that our best approximation appears to account for the symmetric "shoulders" appearing in the experimental data. We take this as the first evidence of the necessity of incorporating the effects of deformation in the theory of the high-energy stripping cross sections. And, finally, as a word of caution, we remind the reader that the experimental data represent a much more complex situation: they are inclusive and an average over intrinsic and rotational excitations with isospin 1 and 2 and up to $10 \mathrm{MeV}$ excitation energy.

Below we show that there are other cases when the effects of deformation are more significant. 


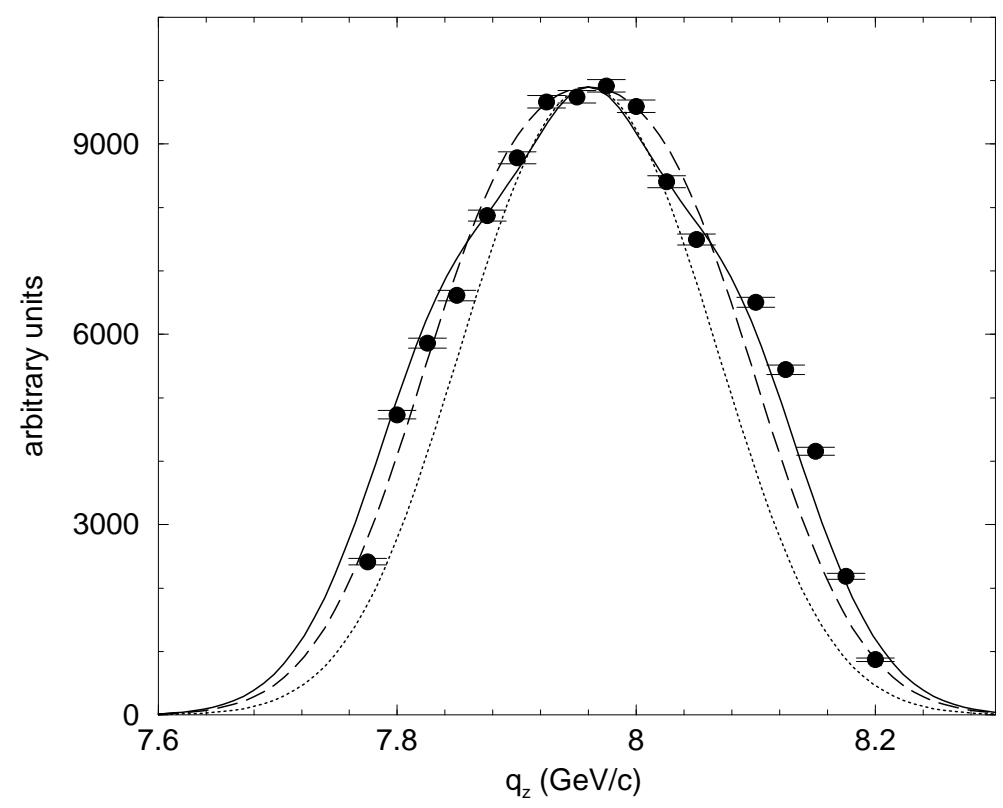

FIG. 4. Longitudinal momentum distributions of ${ }^{24} \mathrm{Mg}$ in the stripping reaction ${ }^{9} \mathrm{Be}\left({ }^{25} \mathrm{Al},{ }^{24} \mathrm{Mg}\right) \mathrm{X}$ calculated in the framework of three different models: (i) the exact theory of Sec. I, solid curve; (ii) the extended geometric approach of Sec. II, dotted curve; (iii) the geometric approach for spherical projectiles of Ref. [9], dashed line. The experimental points together with the error bars from [7] are shown. The initial beam energy is $65 \mathrm{MeV}$ per nucleon.

Let us take as a projectile a nucleus, such as ${ }^{28} \mathrm{P}$, which has the valence proton in the [211 1/2] Nilsson orbital. In reality, the ${ }^{28} \mathrm{P}$ nucleus is spherical or very weakly deformed but we use this example here to illustrate possible deformation effects. The longitudinal momentum distributions for ${ }^{27} \mathrm{Si}$ in the ground state calculated with eq. (20) are shown in Fig. 5 for five values of the deformation parameter $\delta$. 


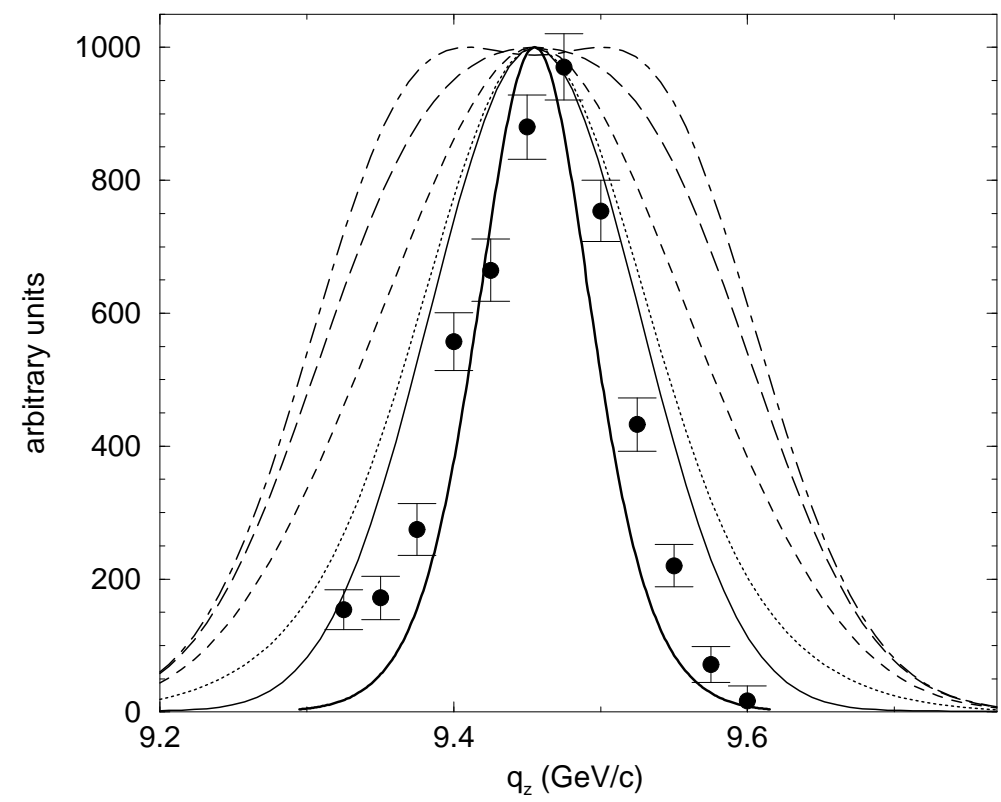

FIG. 5. Longitudinal momentum distributions of ${ }^{27} \mathrm{Si}$ in the stripping reaction ${ }^{9} \mathrm{Be}\left({ }^{28} \mathrm{P},{ }^{27} \mathrm{Si}\right) \mathrm{X}$ with the ${ }^{27} \mathrm{Si}$ nucleus in the intrinsic ground state. The deformation values are $\delta=0$ for the oscillator single-particle wave function (wide solid curve), $\delta=0.1$ (dotted), $\delta=0.2$ (dashed), $\delta=0.3$ (long dashed), and $\delta=0.4$ (dash-dotted). The fat solid curve corresponds to a spherical projectile. It is calculated [7] for a Woods-Saxon potential including the Coulomb interaction which gives the correct asymptotics of the tail of the nucleon wave function. All theoretical curves are normalized to the experimental data which together with error bars are taken from ref. [7]. The experimental data in this case have been corrected for contributions to excited states and represent the branch to the ${ }^{27} \mathrm{Si}\left(5 / 2^{+}\right)$ground state alone. The corresponding partial cross section is $21 \pm 5 \mathrm{mb}$ [7]. The initial beam energy is $65 \mathrm{MeV}$ per nucleon.

It is clearly seen that the width of the longitudinal momentum distributions increases with increasing projectile deformation. The origin of this behavior is the redistribution of the strength of different stretched spherical components in the static field of the deformed core, see Fig. 6. 


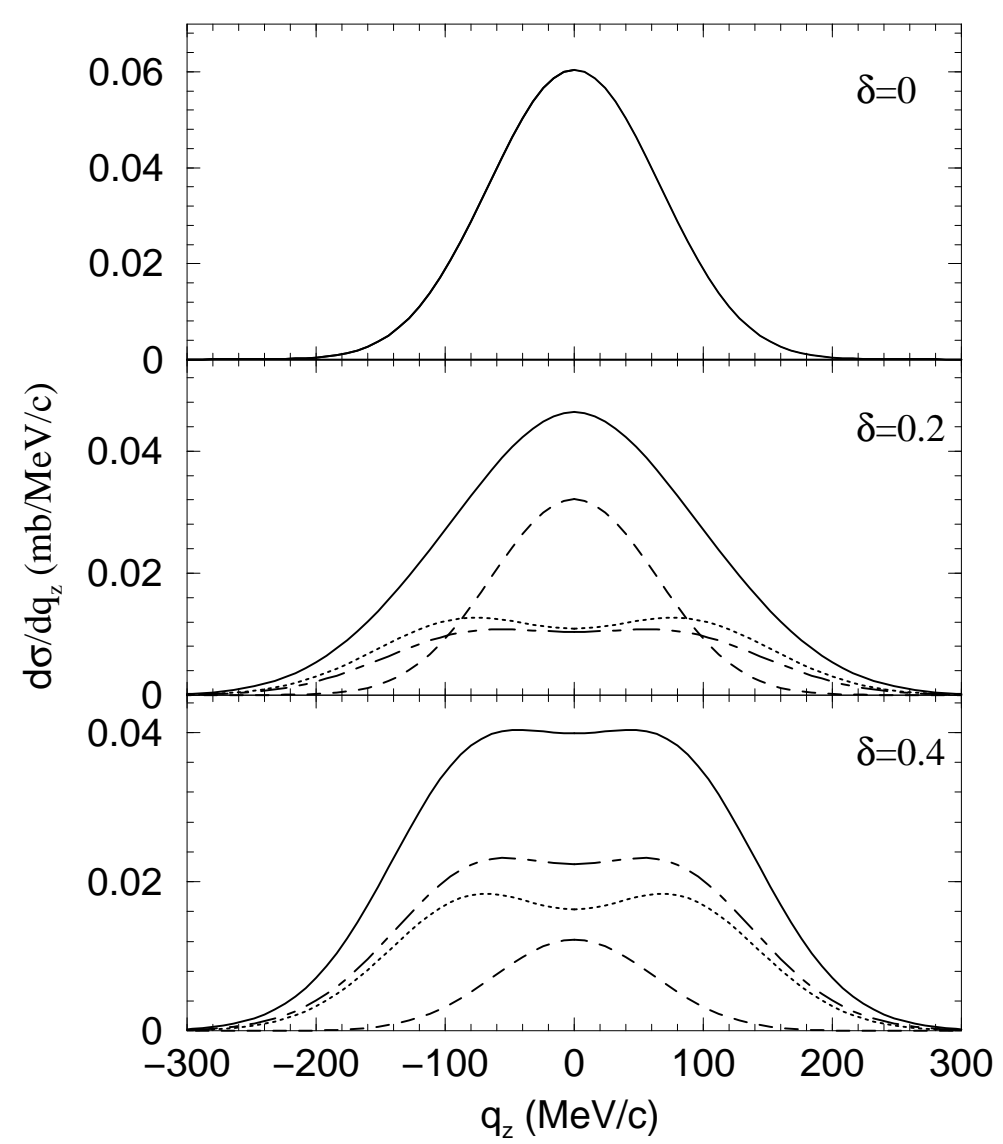

FIG. 6. The total and partial longitudinal momentum distributions of ${ }^{27} \mathrm{Si}$ in the stripping reaction ${ }^{9} \mathrm{Be}\left({ }^{28} \mathrm{P},{ }^{27} \mathrm{Si}\right) \mathrm{X}$ with the ${ }^{27} \mathrm{Si}$ nucleus in the intrinsic ground state, as a function of the assumed deformation values: $\delta=0$ for the upper panel, $\delta=0.2$ for the middle panel, and $\delta=0.4$ for the lower panel. The longitudinal momentum distributions are shown for the proton from different spherical orbitals, $d_{5 / 2}$ (dotted), $s_{1 / 2}$ (dashed), and $d_{3 / 2}$ (dash-dotted); solid curves correspond to the total distribution. At the absence of the deformation all curves coincide (upper panel). All data are shown in the projectile rest frame, so that the momentum scale is different from that in Figs. 2-5.

At small deformations, Fig. 6(b), the $s_{1 / 2}$-wave is dominant but as the deformation increases, Fig. 6(c), the $d_{5 / 2^{-}}$and especially $d_{3 / 2^{-}}$wave overcome, and as a result the shape of the momentum distributions undergoes essential changes. It makes it difficult (if at all possible) to determine the value of the orbital angular momentum of the stripped nucleon from the shape of the core longitudinal momentum distribution. At the same time these 
changes allow one to estimate the value of the deformation parameter $\delta$. We need to bear in mind that the oscillator hamiltonian tends to underestimate the spatial tail of single-particle wave functions. This leads to a wider momentum distribution compared with asymptotically correct wave functions when we use the normalization to the experimental data. This is illustrated by Fig. 5 where the fat solid line corresponds to the asymptotically correct spherical $(\delta=0)$ wave finction. The corresponding total stripping cross section is close to 13 $\mathrm{mb}$ which is considerably less than the experimental value $\sigma=21 \pm 5 \mathrm{mb}$. The ratio of two cross sections, calculated for the oscillator wave functions and for the functions with correct asymptotic behavior, remains nearly constant in all our calculations. The wider solid line is related to the oscillator wave function for the spherical case. The corresponding stripping cross sections are shown in Table 3.

Table 3. Cross sections (in $\mathrm{mb}$ ) for the stripping of the $[2111 / 2]$ proton from ${ }^{28} \mathrm{P}$ at different deformation parameters $\delta$ calculated with harmonic-oscillator wave functions.

\begin{tabular}{|c|c|c|c|c|c|}
\hline$\delta$ & 0 & 0.1 & 0.2 & 0.3 & 0.4 \\
\hline$\sigma$ & 10.5 & 11.0 & 11.7 & 12.8 & 13.4 \\
\hline
\end{tabular}




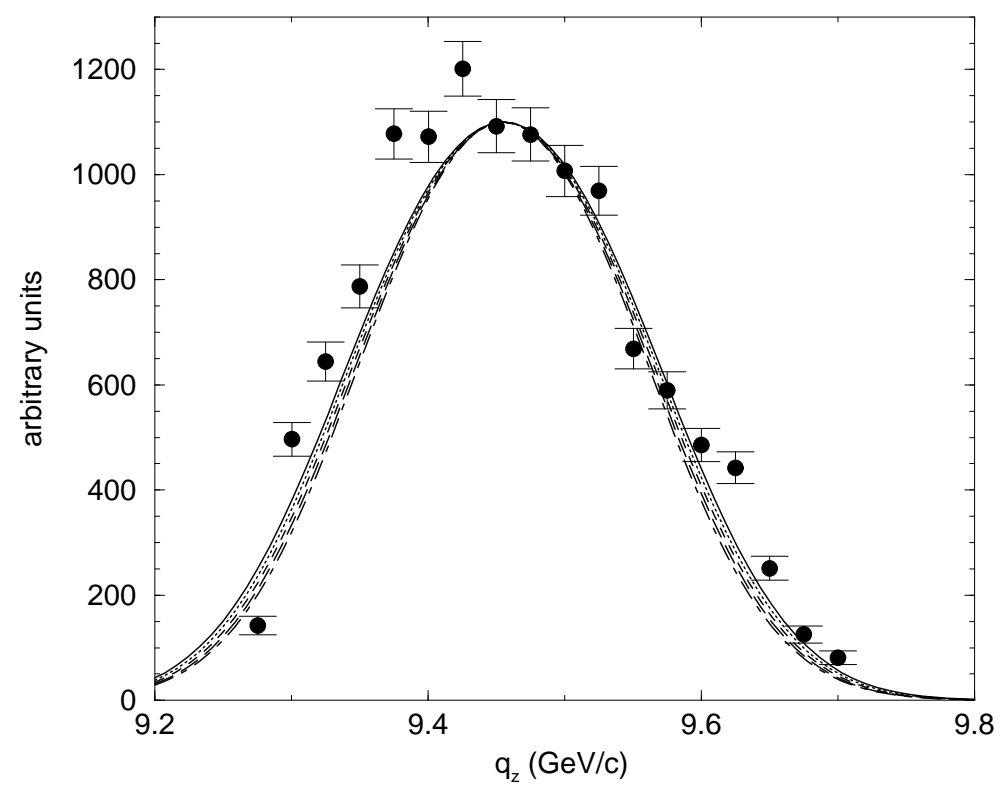

FIG. 7. Longitudinal momentum distributions of ${ }^{27} \mathrm{Si}$ for the hypothetical case of the stripping reaction ${ }^{9} \mathrm{Be}\left({ }^{28} \mathrm{P}^{27} \mathrm{Si}^{*}\right) \mathrm{X}$ with the proton knocked out from the [202 5/2] orbital; the calculations are based on eq. (20). The deformation parameters are $\delta=0$ (solid curve), $\delta=0.1$ (dotted), $\delta=0.2$ (dashed), $\delta=0.3$ (long dashed), and $\delta=0.4$ (dash-dotted). The experimental points together with errors from [7] are shown. The initial beam energy is $65 \mathrm{MeV}$ per nucleon.

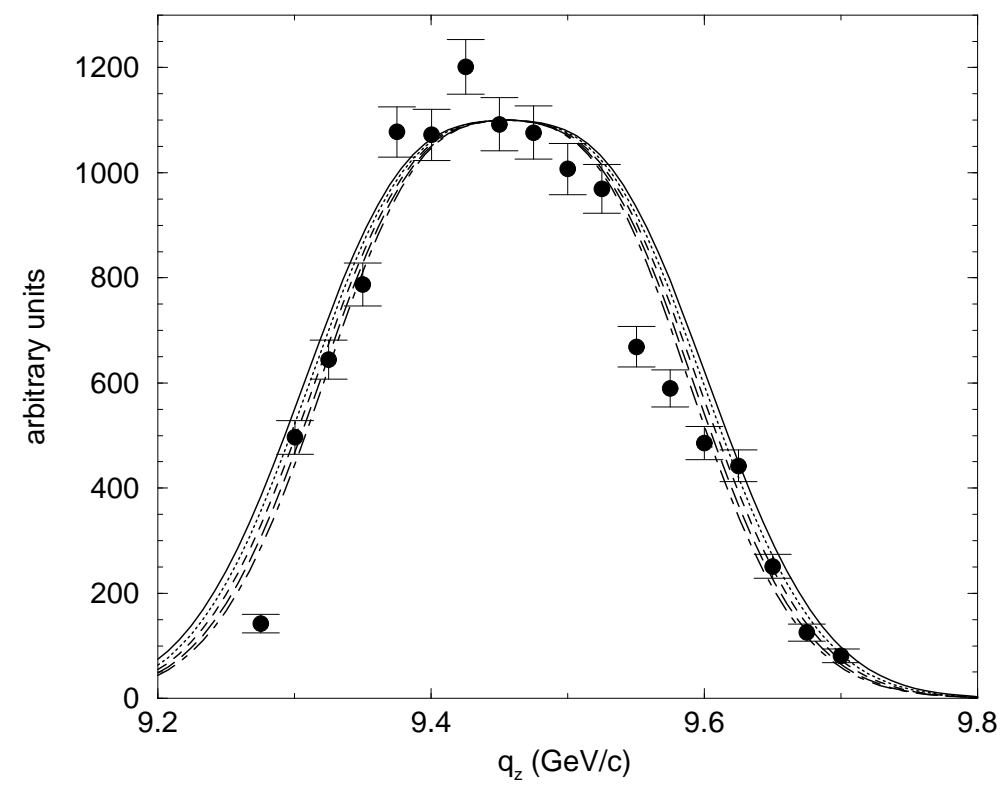

FIG. 8. The same as Fig. 7, for the stripping from the [211 3/2] orbital. 
Table 4. The cross sections (in mb) for the stripping of [202 5/2], [211 3/2], and [220 1/2] protons from ${ }^{28} \mathrm{P}$ at different deformation parameters $\delta$.

\begin{tabular}{|c|c|c|c|c|c|}
\hline$\delta$ & 0 & 0.1 & 0.2 & 0.3 & 0.4 \\
\hline$\sigma$ for $[2025 / 2]$ & 17.1 & 16.5 & 16.1 & 16.0 & 16.2 \\
\hline$\sigma$ for $[2113 / 2]$ & 16.5 & 15.9 & 15.7 & 15.7 & 16.0 \\
\hline$\sigma$ for $[2201 / 2]$ & 22.4 & 20.6 & 19.2 & 17.9 & 18.5 \\
\hline$\sum \sigma$ & 55.9 & 53.0 & 52.4 & 49.7 & 50.7 \\
\hline$\sum \sigma+\sigma$ for $[2111 / 2]$ & 66.4 & 64.0 & 64.1 & 62.5 & 64.1 \\
\hline
\end{tabular}

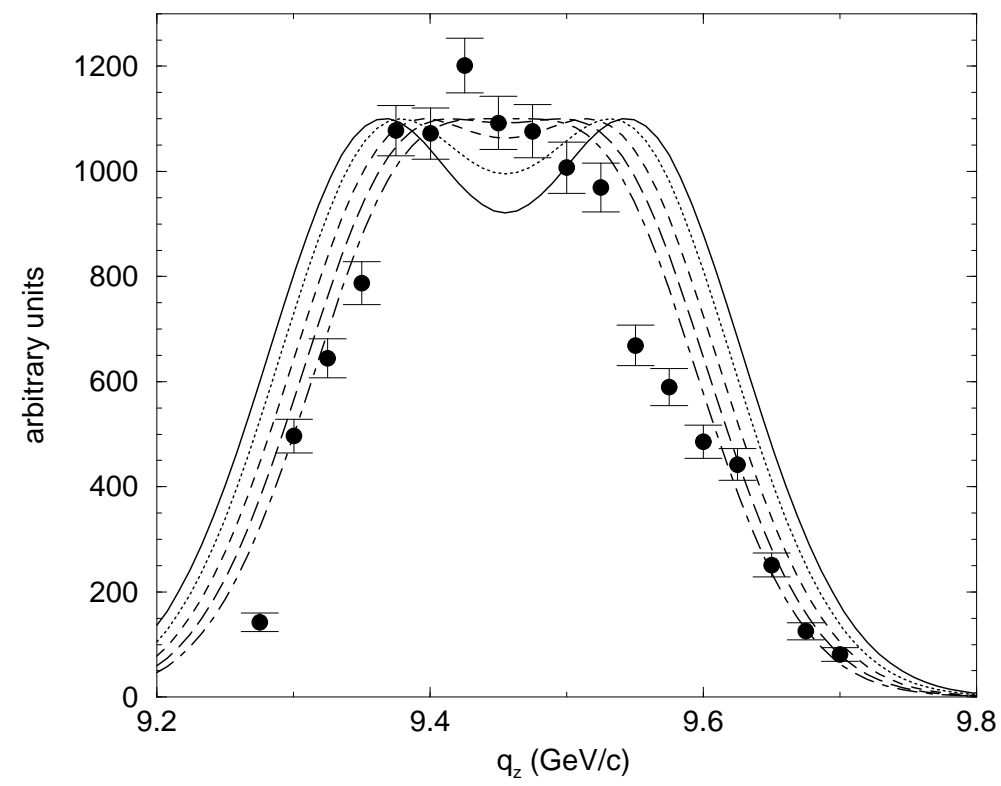

FIG. 9. The same as Fig. 7, for the stripping from the [220 1/2] orbital.

One of the attractive features of our simple approach is the possibility to treat the stripping reactions with the core excitation in the same manner. For example, for the particular reaction ${ }^{9} \mathrm{Be}\left({ }^{28} \mathrm{P},{ }^{27} \mathrm{Si}^{*}\right) \mathrm{X}$ with the detection of the excited projectile residue ${ }^{27} \mathrm{Si}^{*}$, we have three orbitals in the $s d$-shell, [202 5/2], [211 3/2], and [220 1/2]. Assuming that the measured [7] cross section is originated by the knockout from an individual orbital we 
obtain the longitudinal momentum distributions shown in Figs. 75. For the comparison with experimental data, $\sigma=70 \pm 11 \mathrm{mb}$, we have to sum the partial cross sections because the different core final states were not separated in the experiment [7]. The corresponding values are shown in the last row of Table 4 including the contribution from the ground state orbital [211 1/2] given in Table 3. In the treatment with the use of the spherical shell model [7] it is important to include the relevant spectroscopic factors. In the simple Nilsson model used here, we assume that pairing is weak and that the occupation numbers simply take the values $(0,1,2)$. We do not take into account the relevant spectroscopic factors which depend on the deformation of the core mean field and can not be merely borrowed from the standard shell-model calculations. To make our consideration fully quantitative, we would need also (i) to correct the asymptotic behavior of harmonic oscillator wave functions, (ii) to take into account feeding from high-lying states [9]. Nevertheless, even oversimplified estimations of the partial and total cross sections, Table 4, show a relatively weak dependence on deformation whereas the shape of the longitudinal momentum distributions, Figs. 779, is more sensitive to the deformation parameter.

Based on those results we may try to predict the shape of the longitudinal momentum distributions of the projectile residue in reactions with strongly deformed nuclei. As a possible candidate for the role of the deformed projectile we can use neutron-rich ${ }^{26,28} \mathrm{Ne}$ or ${ }^{30,32,34} \mathrm{Mg}$ isotopes. The measurements and analysis of recent work [16] allow one to expect a large deformation of these nuclei. Therefore, it would be interesting to study the longitudinal momentum distributions of the projectile residue, for example in the reaction ${ }^{9} \mathrm{Be}\left({ }^{30} \mathrm{Mg},{ }^{29} \mathrm{Mg}_{\text {g.s. }}\right) \mathrm{X}$. The preliminary estimate based on the measurement of the reduced transition probability $B\left(E 2 ; 0_{\text {g.s. }}^{+} \rightarrow 2^{+}\right)$gives $\delta \approx 0.46-0.52$ for the deformation parameter of the projectile. It corresponds to the knockout of a neutron either from [321 3/2] or from [202 5/2] Nilsson orbitals (here we extrapolate the appropriate Nilsson diagram to large deformations). The first orbital comes out from the $p f$-shell (intruder) whereas the second one belongs to the $s d$-shell. The orbitals intersect just in the region of $\delta$ indicated by the experiment [16]. Thus, we obtain an interesting possibility to determine actual deformation 
of ${ }^{30} \mathrm{Mg}$ via the comparison with experimental data and clarify the internal structure of this nucleus. Similar arguments are true for other isotopes in this region. To illustrate this possibility we have calculated, see Fig. 10, the longitudinal momentum distributions for the neutron knocked out from [202 5/2] Nilsson orbital, solid curve, and from the intruder orbital [321 3/2], dashed curve. Two results differ very strongly both in the magnitude of the cross section and, which is even more essential, in shape. The estimate of the corresponding total cross sections gives $\approx 8 \mathrm{mb}$ for the $[2025 / 2]$ and $\approx 16 \mathrm{mb}$ for the [321 3/2] orbital.

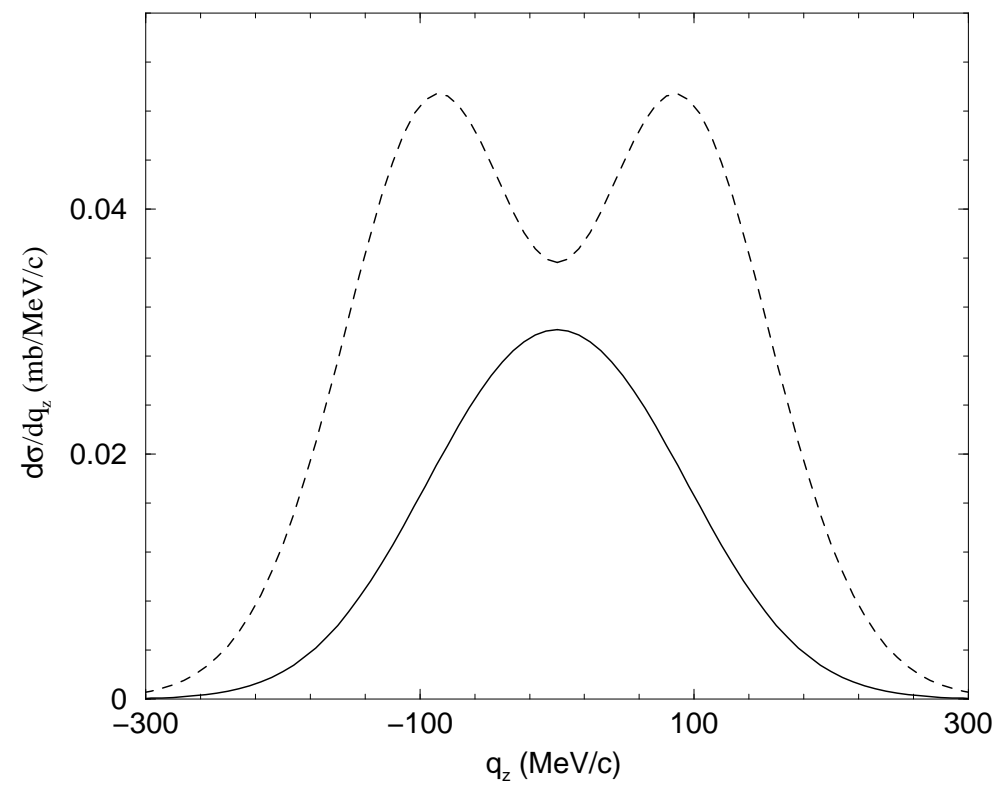

FIG. 10. Longitudinal momentum distributions of ${ }^{29} \mathrm{Mg}$ in the stripping reaction ${ }^{9} \mathrm{Be}\left({ }^{30} \mathrm{Mg},{ }^{29} \mathrm{Mg}\right) \mathrm{X}$ with the ${ }^{29} \mathrm{Mg}$ nucleus in the intrinsic ground state. The neutron is knocked out from the [202 5/2] orbital, solid curve, and from the intruder [321 3/2], dashed curve. The deformation parameter for both curves is $\delta=0.5$. The distributions are calculated in the projectile rest frame.

\section{CONCLUSION}

We investigated the influence of the projectile deformation on stripping cross sections and longitudinal momentum distributions of the core. For this purpose we developed an appropriate formalism treating the deformed projectile mean field as in the standard Nilsson model. In practical calculations, we limited ourselves by the fast processes with the 
core nucleus as a spectator its orientation being adiabatically fixed during the short collision time. The study of reactions like ${ }^{9} \mathrm{Be}\left({ }^{25} \mathrm{Al},{ }^{24} \mathrm{Mg} g . s\right) \mathrm{X},{ }^{9} \mathrm{Be}\left({ }^{28} \mathrm{P},{ }^{27} \mathrm{Si}_{\text {g.s. }}\right) \mathrm{X}$, and ${ }^{9} \mathrm{Be}\left({ }^{28} \mathrm{P},{ }^{27} \mathrm{Si}^{*}\right) \mathrm{X}$ shows the following.

(i) The general influence of the deformation on the stripping cross section and the core longitudinal momentum distribution is relatively weak for the knockout of nucleons from orbitals which are not, or are only slightly, mixed by the deformed mean field of the projectile. Nevertheless, even in such cases it is possible to reproduce the details of the experimental longitudinal momentum distributions only taking into account the deformation effects.

(ii) The deformation of the projectile is seen the most clearly in the knockout of nucleons from the strongly mixed orbitals. In the case of the axial symmetry it corresponds to the lowest possible $\Omega$.

(iii) The influence of deformation may be less pronounced in the case of halo systems where the wave function of the valence nucleon is extended far outside the core. The choice of ${ }^{11} \mathrm{Be}$ as a projectile 42] seems to be a good testing ground for verifying this conclusion.

(iv) The use of harmonic-oscillator wave functions with a wrong asymptotic behavior at large distances is quite meaningful for a projectile with high nucleon separation energy.

(v) The geometric approach to the description of the stripping reactions is quite applicable for an estimation of the reaction cross sections but it is oversimplified for reproducing the longitudinal momentum distributions where we need to use a more detailed semi-microscopical treatment.

(vi) The developed in Seq. II semi-microscopic approach appears to account for the symmetric "shoulders" of the longitudinal momentum distributions appearing in the experimental data. We take this as the first evidence of the necessity of incorporating the effects of deformation in the theory of the high-energy stripping reactions.

(vii) The analysis of the shape of the core longitudinal momentum distribution allows one to estimate the degree of the projectile deformation. This is necessary for determining the internal structure of radioactive nuclei. The region of neutron-rich heavy magnesium isotopes would be a promising field for future experiments. 
(viii) It would be interesting to demonstrate in further calculations the predicted by a simpler approach difference in the momentum distributions of a strongly deformed core for the processes leading to the individual members of a rotational band.

(ix) In the case of the deformed projectile it is impossible to neglect the spin-orbit interaction since it defines the single-particle orbitals in the deformed core mean field.

(x) An analysis of experimental data ignoring the effects of the deformation can lead to significant errors in assigning orbital angular momentum involved in the stripping reaction.

\section{ACKNOWLEDGMENTS}

We are thankful to J.A. Tostevin for constructive discussions and reading the manuscript, and to A. Navin for discussion of experimental data. This work was supported by the National Science Foundation, through Grant 96-05207.

[1] S.T. Butler, Nuclear Stripping Reactions (Wiley, New York, 1957).

[2] A.G. Sitenko, Theory of Nuclear Reactions (World Scientific, Singapore, 1990).

[3] R. Serber, Phys. Rev. 72, 1008 (1947).

[4] I. Tanihata, Prog. Part. Nucl. Phys. 35, 505 (1995).

[5] F. Barranco, E. Vigezzi, and R.A. Broglia, Z. Phys. A 356, 45 (1996).

[6] P.G. Hansen, J. Phys. G 25, 727 (1999).

[7] A. Navin, D. Bazin, B.A. Brown, G. Gervais, T. Glasmacher, K. Govaert, P.G. Hansen, M. Hellström, R.W. Ibbotson, V. Maddalena, B. Pritychenko, H. Scheit, B.M. Sherill, M. Steiner, J.A. Tostevin, and J. Yurkon, Phys. Rev. Lett. 81, 5089 (1998).

[8] J.A. Tostevin, J. Phys. G 25, 735 (1999).

[9] P.G. Hansen, Phys. Rev. Lett. 77, 1016 (1996). 
[10] K. Hencken, G. Bertsch, and H. Esbensen, Phys. Rev. C 54, 3043 (1996).

[11] F. Barranco and E. Vigezzi, In: International School of Heavy-Ion Physics, 4th Course: Exotic Nuclei, edited by R.A. Broglia and P.G. Hansen (World Scientific, Singapore, 1998), p. 217.

[12] B.H. Wildenthal and W. Chung, Phys. Rev. C 22, 2260 (1980).

[13] K. Heyde and J.L. Wood, J. Phys. G 17, 135 (1991).

[14] E. Caurier, F. Nowacki, A. Poves, and J. Retamosa, Phys. Rev. C 58, 2033 (1998).

[15] E.K. Warburton, J.A. Becker and B.A. Brown, Phys. Rev. C 41, 1147 (1990).

[16] B.V. Pritychenko, T. Glasmacher, P.D. Cottle, M. Fauerbach, R.W. Ibbotson, K.W. Kemper, V. Maddalena, A. Navin, R. Ronningen, A. Sakharuk, H. Scheit, and V.G. Zelevinsky, Phys. Lett. B, to be published.

[17] D. Ridikas, M.H. Smedberg, J.S. Vaagen, and M.V. Zhukov, Nucl. Phys. A 628, 363 (1998).

[18] J.A. Christley and J.A. Tostevin, Phys. Rev. C 59, 2309 (1999).

[19] A. Bohr and B.R. Mottelson, Nuclear Structure, Vol. 2 (Benjamin Inc., New York, 1974).

[20] S.I. Drozdov, Sov. Phys. JETP 1, 588, 591 (1955); 7, 889 (1958).

[21] E.V. Inopin, Sov. Phys. JETP 3, 134 (1956).

[22] Y. Abgrall, J. Labarsouque, and B. Morand, Nucl. Phys. A316, 389 (1979).

[23] G. Fäldt and R. Glauber, Phys. Rev. C 42, 395 (1990).

[24] G. Fäldt, J. Phys. G 6, 1513 (1980).

[25] H. Nishioka and R.C. Johnson, J. Phys. G 8, 39 (1982).

[26] K. Yabana, Y. Ogawa, and Y. Suzuki, Nucl. Phys. A 539, 295 (1992).

[27] T. Fujita and J. Hüfner, Nucl. Phys. A343, 493 (1980). 
[28] J.S. Al-Khalili, I.J. Thompson, and J.A. Tostevin, Nucl. Phys. A 581, 331 (1995).

[29] R.J. Glauber, In: Lectures in Theoretical Physics, edited by W.E. Brittin and L.G. Dunham (Interscience, New York, 1959), Vol. I, p. 315.

[30] R.J. Glauber and G. Matthiae, Nucl. Phys. B21, 135 (1970).

[31] A.I. Akhieser and A.G. Sitenko, Phys. Rev. 106, 1236 (1957).

[32] M.S. Hussein and K.W. McVoy, Nucl. Phys. A445, 124 (1985).

[33] M.S. Hussein and R.C. Mastroleo, Nucl. Phys. A491, 468 (1989).

[34] P. Banerjee and R. Shyam, Nucl. Phys. A 561, 112 (1993).

[35] A. Bohr and B.R. Mottelson, Nuclear Structure, Vol. 1 (Benjamin Inc., New York, 1969).

[36] E.K. Warburton and B.A. Brown, Phys. Rev. C 46, 923 (1992).

[37] J. Joachain, Quantum Collision Theory (North-Holland, Amsterdam, 1980).

[38] S.G. Nilsson, Kgl. Danske Vidensk. Selsk. Mat.-Fys. Medd. 29, No. 1 (1955).

[39] S.G. Nilsson and I. Ragnarsson, Shapes and Shells in Nuclear Structure (Cambridge University Press, Cambridge, 1995).

[40] A.R. Edmonds, Angular Momentum in Quantum Mechanics (Princeton University Press, Princeton, 1960).

[41] E.W. Lees, C.S. Curran, T.E. Drake, W.A. Gillespie, A. Johnston, and R.P. Singhal, J. Phys. G 2, 105, (1976).

[42] T. Aumann et al., to be published. 